\title{
An Efficient Generic Review Framework for Assessment of Learners Ability using KNN Algorithm
}

\author{
Saravanakumar $\mathrm{C}^{1}$, Geetha $\mathrm{M}^{2}$, Govindaraj $\mathrm{V}^{3}$, Prakash Mohan ${ }^{4}$, Vijayakumar $\mathrm{K}^{5}$ \\ \{mailofcsk@gmail.com¹, geetham1@srmist.edu.in², govicse15@gmail.com ${ }^{3}$, \\ salemprakash@gmail.com ${ }^{4}$,mkvijay@msn.com ${ }^{5}$ \} \\ ${ }^{1}$ Associate Professor, Department of Information Technology, \\ St.Joseph's Institute of Technology, OMR, Chennai - 600119, India \\ ${ }^{2}$ Professor, Department of Computer Science and Engineering, School of Computing, \\ SRM Institute of Science and Technology, Vadapalani, Chennai, India, \\ ${ }^{3}$ Assistant Professor, Department of Information Technology, \\ St.Joseph's Institute of Technology, OMR, Chennai - 600119, India \\ ${ }^{4}$ Professor, Department of Computer Science and Engineering, \\ Karpagam College of Engineering, Coimbatore, India \\ ${ }^{5}$ Associate Professor, Computer Science and Engineering, \\ St.Joseph's Institute of Technology, OMR, Chennai - 600119, India
}

\begin{abstract}
Nowadays online review and recommendation system plays major role for maintaining quality of any kind of product with various domain. The customer has to evaluate the product and provides the positive as well as negative reviews based on their interest. The education domain has various stages of the evaluation namely staff related, student related and organization related and so on. Normally the review is restricted to the particular college or university because of their own protocols and system. The proposed framework is to implement the generic review system based on the respective configuration. There are various templates introduced to get the accurate review with good evaluation result. This framework is modeled using the layer of abstraction. User interface layer provides the complete support for the user who uses the system with customized design. Review layer handles all data in different by retrieving and storing the credentials for further review process. Application configuration layer is used to provide the template for assigning credentials and authorization with complete configuration of the system. The final reports are taken for the evaluation process for taking corrective action for further improvement. The classification of the review is carried out in order to achieve high level of accuracy. The main objective of the proposed framework is to classify the student review using KNN (K Nearest Neighbor) algorithm with high efficiency.
\end{abstract}

Keywords: Cloud Computing, Edge Computing, Scheduling, Internet-of-Things, Data Analytics..

\section{Introduction}

Nowadays the online review and evaluation process vital role in all domain because of maintaining maximum quality and improve the outcome based on the customer suggestion. In marketing domain needs the customer review for improving the sales of any product which is in any form. Online social networking application needs maximum customer review for 
satisfying the customer in an extreme level. If they unable to meet the customer feedback, then they are in the position of loss the valuable customer. This is also applicable in the education domain the review and analysis is carried out various forms with different parameters, so that the academic institution achieves maximum quality outcome. Latent semantics analysis (LSA) is a technique is used to classify the reviewer's comments in high quantity and quality manner. K Nearest Neighbor algorithm is used to classify the items which are close to each other. It uses the features like distance measures, proximity measures and closeness measures. The outcome of the algorithm is based on the number of clusters which are selected during the initial condition.

The machine learning algorithms are used for this purpose. The review has been done by performing various preprocessing stages with meta based review comments. The comments are classified using text classification algorithm which are given by the students about the institution [1]. The student reviews about the college and staff members are analyzed and improved based on the comments either oral or written. The aim of the review is to satisfy the customers for the expected level. STEM is education methodology is used to identify the presence and absence of the quality in the education system. The online reviews are collected from various university around the world from the students in order to analyze the sentiments when compared to the traditional analysis [2].

Information technology based job needs the training based the categories such as construction of ideas, interaction and collaboration, communication ability, high level programming skills in order to develop application in order to solve the complex problems. The selection of the training centers and types needs reviews from various persons with parameters. The student has to analyze the eco system in IT is carried out by using the effective online evaluation system [3]. The problem faced by the teacher as well as professor has difficult to give the review regarding the software project in a current trend. The review parameters are comments related to the code and bugs, accuracy score related to the project in all dimensions. Two stages of feedback have been conducted for handling unbiased review by the student.

The automatic tested is conducted in order to assess the final grade in the project rank [4]. The review about the projects which are created by the students with the features like strength and weakness related to the development. The student performances are compared with the other users in order to assess the learning process and its method. The monitoring of the technical and issues and results are done for further quality improvement [5]. Group based ranking information has been done by considering the comments of the users by the peer students in the college. There are three levels of the review stages namely selection of the review protocol, two stage review process and peer selection. Information are provided by the students based on the conditions with additional features [6].

The peer reviews process is not efficient because of the heavy workload allocated to the staff members as well as students. This problem is overcome by implementing the methods to the users and staff members. Classification based analysis is carried out in order to achieve the high review evaluation score. The results are represented as graph, data and figures with visualization for better understanding of review comments and assessment. The analysis is done with longer comments, more users in order to reduce the teacher's workload [7]. 
Principal component analysis is used to evaluate the college quality based on the information given by the students.

The evaluation rank as well as index is calculated in higher dimensional manner. The aim of the proposed method of assessment is to enhance the quality of the student in the global market [8]. The higher education peer reviews are assessed by using the integrated approach. The information and parameters are used in the feedback system analyzed. The list of parameters is self-assessment, student training skills, group review, specific and generic comments about the staff members and college [9]. Moodle based online training program plays important role in the student skill enhancement process. This system provides the student grade prediction, student and staff research collaboration, association and inters activities of the students. The online quiz is conducted for collecting the data and review comments for calibration [10].

Customer feedback system gives the reliable information about the views in various dimensions in the areas of medical, social networking, review web sites etc. The student opinion about the zig bee devices and its applications are more popular because of sharing of the feedback and related information. There are various API available for write the reviews in the text based sentiment analysis. These data are classified and clustered using efficient machine learning algorithms [11]. Course satisfaction review is given by the students in an automated manner with various visualization methods. This type of visualization gives the better understanding of the teaching and learning process.

The text based comments are not clear so need a better mechanism to do the evaluation process. Machine learning algorithms are supported by analyze and visualization in order to reveal the sentiments in the form of summary [12]. The existing methods are related to particular area of review analysis with specific type of organization. This is implemented by apply the generic based review framework which support all kind of domain areas with various configuration features. The main aim of the proposed system is to analyze the generic model for better reliability and efficiency.

\section{Proposed Resource Management Model}

The proposed system focuses on the review and evaluation of the college infrastructure and staff in formation by the students of college and university. There are four layers in this framework namely user interface layer, application configuration layer, review evaluation layer and report generation layer. There are three types of users needed for interaction namely student, admin and authority.

Students have to provide various parameters regarding the college, staff and other parameters. Traditional technique for taking assessment and review is restricted to only one academic institute, which is not suitable for all types of institutions. This problem is over come by using generic review process, which will help the institution and students for better assessment and evaluation process. 
The student has to login the application by providing unique information which is maintained for all kind of access. This is validated for retrieving suitable information of session initiation. This is generic application so the language selection also considered for interaction by all kind the people in various location and culture. The common information about the institution like logo and name of the institution with relevant information is retrieved from the server for further development. Application configuration layer is used to configure the parameters, which are needed for making the application. The development of the generic review application is carried out using the template because of various institutes are using different parameters for evaluating the staff as well as the institution.
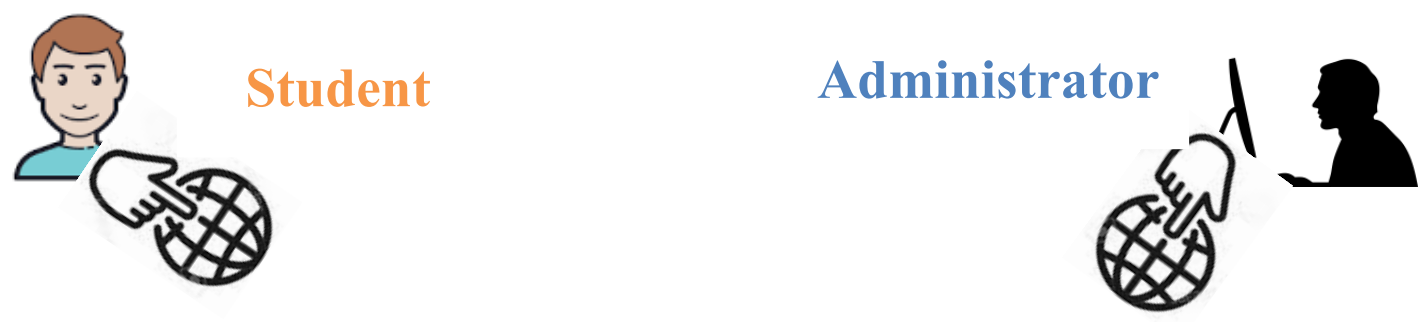

User Interface Layer

Application Configuration Layer

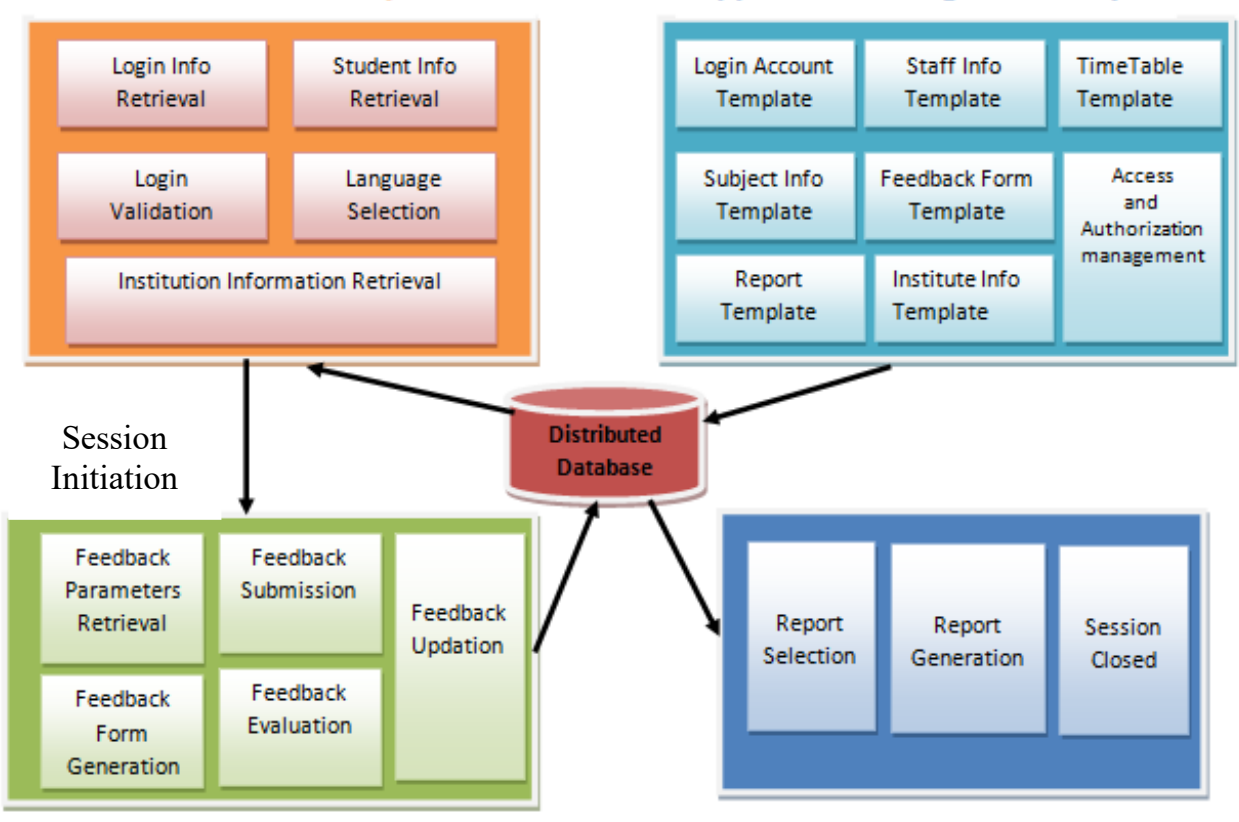

Feedback Evaluation Layer

Report Generation Layer

\section{Authority}

Fig 1 Architecture of the proposed generic review assessment and evaluation model

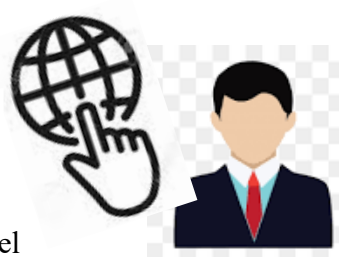


Login account template used for creating list of users need for interaction with relevant features. Staff information template is used to creating the staff both teaching and nonteaching details for evaluation purpose. If the staff is a teaching, then the timetable is uploaded in to the server based on the timetable template. The subject list also configured based on the subject information template. The evaluation parameters are differing from one institute to another so this will be configured using review form template. Report template used to give the different form of report needed for further analysis. Institute information template used to configure the suitable information for further application development. The access and authorization are allocated using the corresponding application access. This process is going to handle and configure by the admin user who secure the entire application from unauthorized access and disclosure.

The review evaluation model is done by retrieving the necessary parameters for respective entry of the assessment. The feedback form is also accessed for making the assessment with proper types and category. The feedbacks are updated in to the distributed database for maintaining consistent information. Report generation layer is used to generate various reports for further evaluation process. There are multiple types of interaction in different rate is handled by using high-end servers with databases. The overall architecture of the proposed generic model is shown in figure 1. The workflow of the generic review application also represented in figure 2 .

\section{Workflow diagram of generic student feedback application}

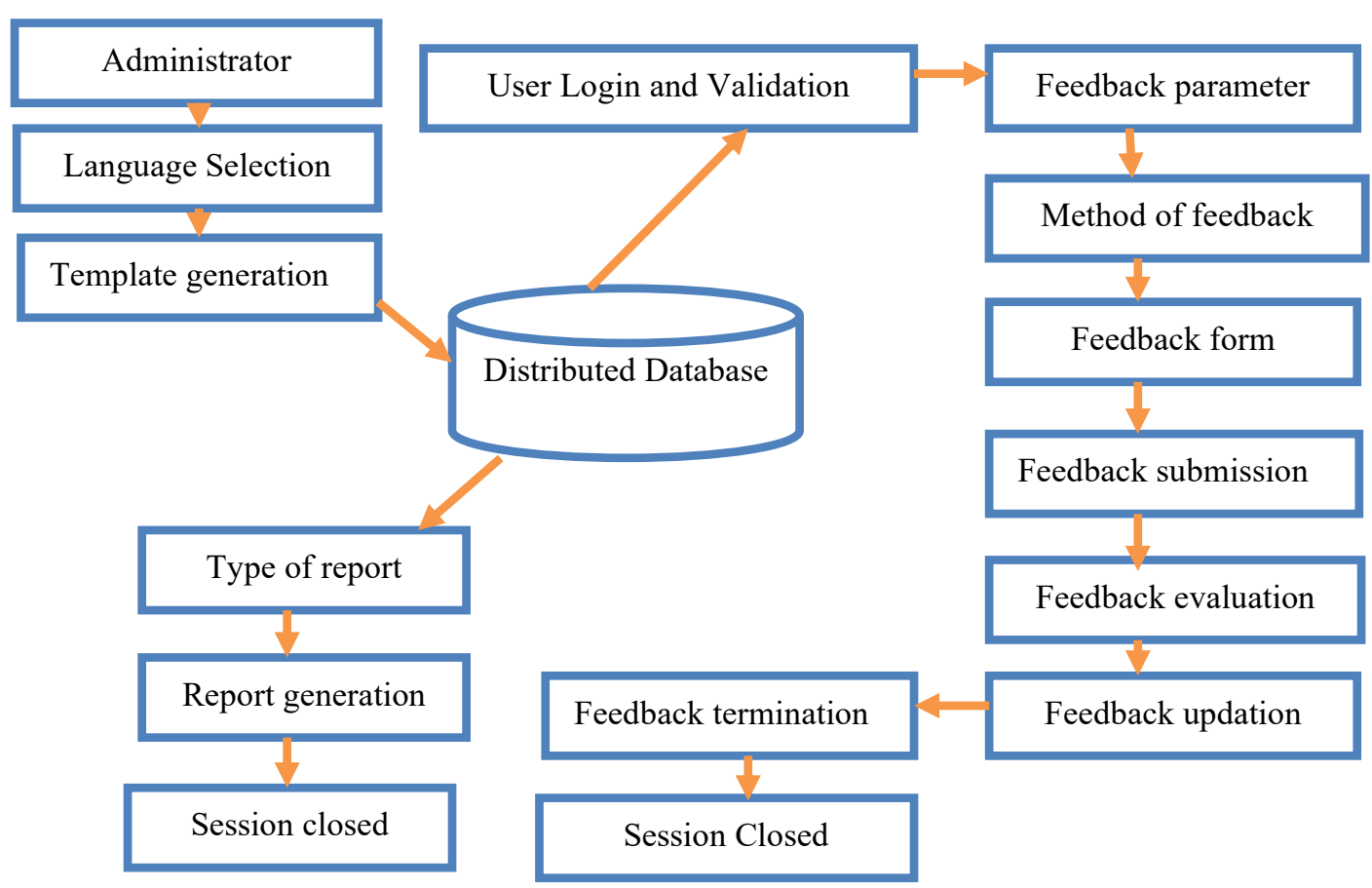


Fig 2Generic student review application

\section{Algorithm}

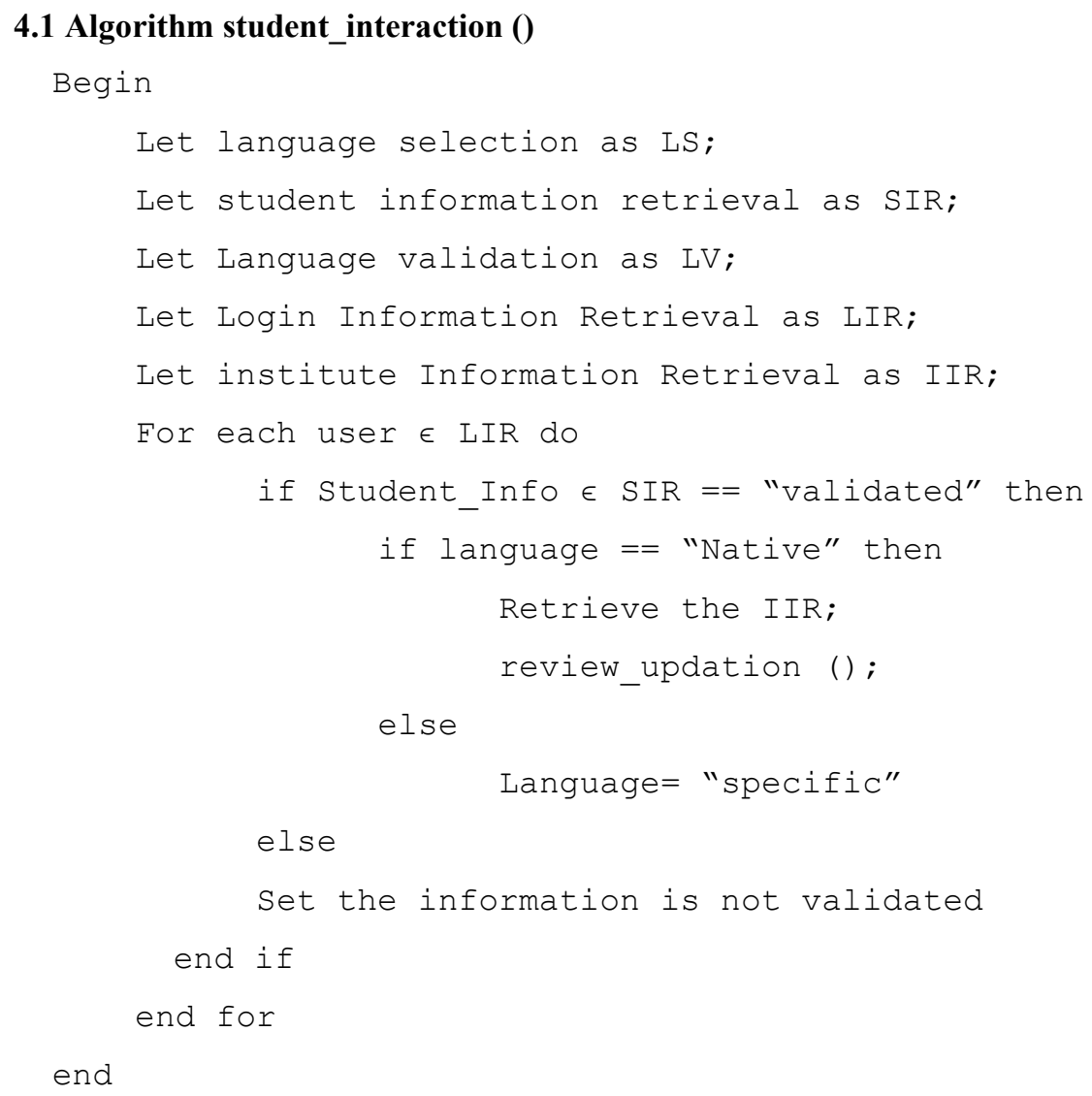

\subsection{Algorithm Review_Updation ()}

Begin

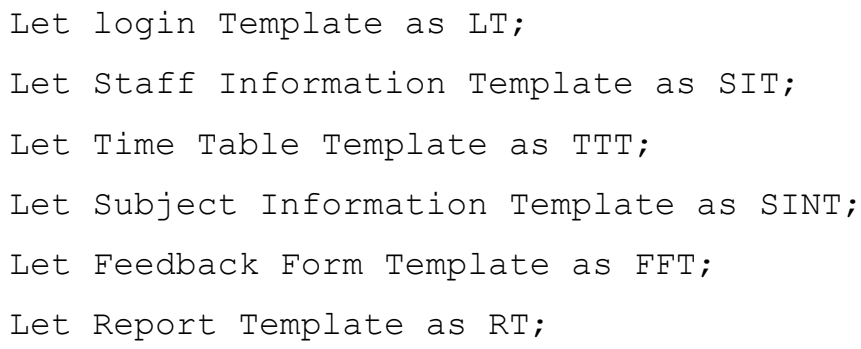




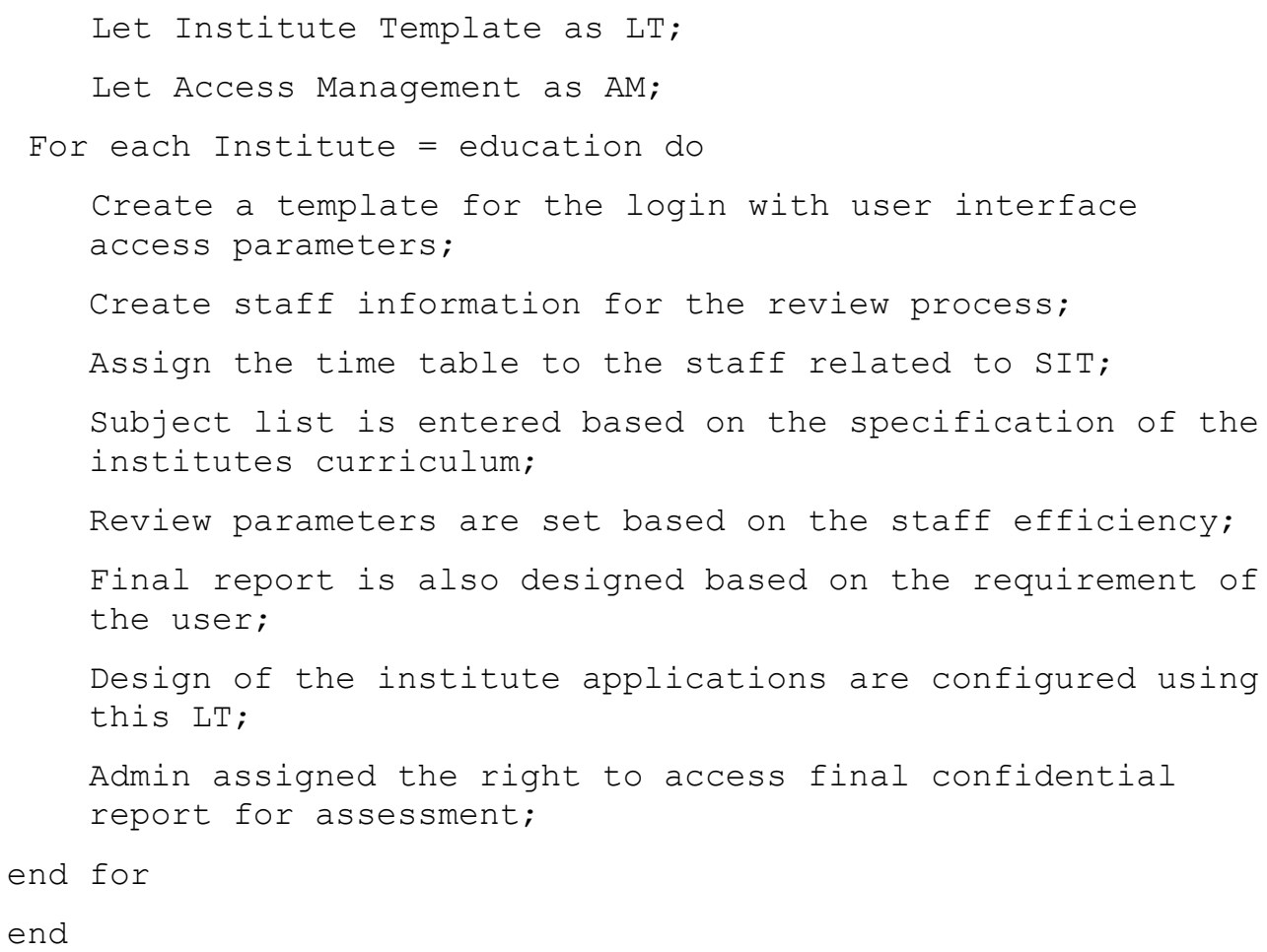

\section{Comparison of various review systems}

The process of identifying and detecting the online reviews and feedbacks done by semi supervised learning method. It also considering the accuracy metrics of classification called FScore with the value of 0.837 . There are various features are identified in order to reduce the classification error. The features are named as speech oriented, word frequency count based, sentiment oriented and so on. This type of system support both positive as well as negative reviews. Normal reviews only considered for making the profit related to any kind of comments, so meta- data based analysis is very vital while handling online reviews from customer [13]. Social media uses the new form of content is called micro review model. The review comments are restricted to 200 characters which suffers the complete review analysis. The data are collected starts from small set of reviews to the larger set of reviews [14]. Hypergraph model is used to embedding the sentiment analysis based on the online review from various resources and formats. The detection of semantic related information in order to extract multidimensional textual features. CNN model used for identifying high order samples and its relations based on the review domain area [15]. The review system needs to perspectives namely vendor profile and reviewer profile. Normally the reviews are taken in to account in blind manner because of irrelevant profiles. Traditional way of representing the reviews are static which suffers various quality issues, so dynamic representation must be used for better accuracy. This is achieved by implementing the fuzzy based clustering with meta data [16] [17]. The above mentioned issues are solved by implementing the novel generic 
based review system with customized template support in order to achieve high performance and efficiency. Table 1 shows that the mapping of dependent features over independent features. Table 2 shows that Correlation Analysis of the class features.

Table 1 Class Mapping of Various Features

Class

Relation raisedhands Vis|TedResources AnnouncementsView

\begin{tabular}{cccccc}
\hline H & 271.140845 & 70.288732 & 78.746479 & 53.380282 & 53.661972 \\
L & 199.527559 & 16.889764 & 18.322835 & 15.574803 & 30.834646 \\
M & 244.540284 & 48.938389 & 60.635071 & 40.962085 & 43.791469
\end{tabular}

Table 2 Class Mapping of Various Features with Correlation Analysis

\begin{tabular}{rrrrrr} 
& & Relation & raisedhands & Vis/TedResources & AnnouncementsView \\
\hline & 1.000000 & 0.999481 & 0.996811 & 0.998840 & 0.997588 \\
Relation & 0.999481 & 1.000000 & 0.993725 & 0.996772 & 0.999306 \\
raisedhands & 0.996811 & 0.993725 & 1.000000 & 0.999497 & 0.988869 \\
Vis/TedResources & 0.998840 & 0.996772 & 0.999497 & 1.000000 & 0.993090 \\
AnnouncementsView & 0.997588 & 0.999306 & 0.988869 & 0.993090 & 1.000000
\end{tabular}

The histogram analysis of the class variable gives the classification accuracy is shown in fig 3 . The feedback are collected based on the dicussion among the students and other stack holders is shown in fig 4. Fig 5 and fig 6 represented Satisfaction based analysis and density based analysis respectively. Relevant attributers and review outcome is presented in fig 7 and fig 8 . The classification accuracy and KNN performance is shown in fig 9 and fig 10 respectively. Table 3 provides the performance and efficiency report of the proposed system. 


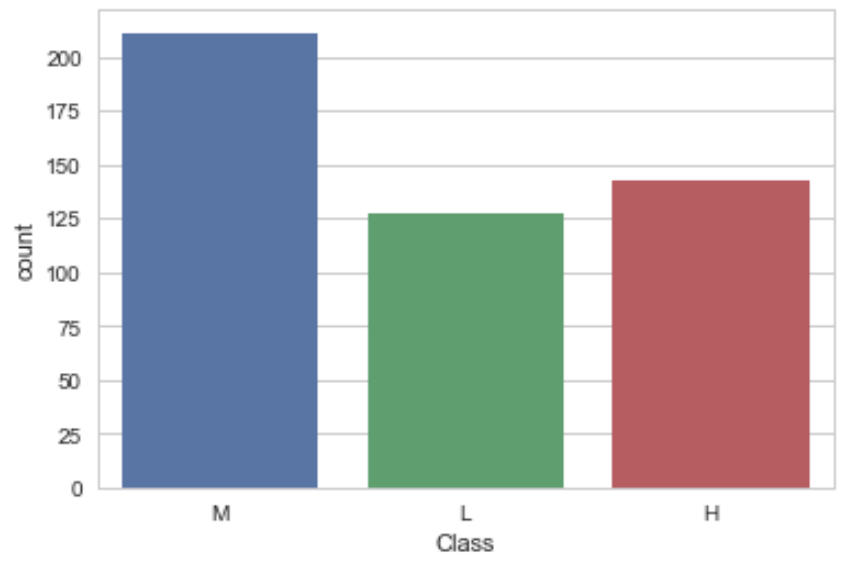

Fig 3 Histogram analysis of Class

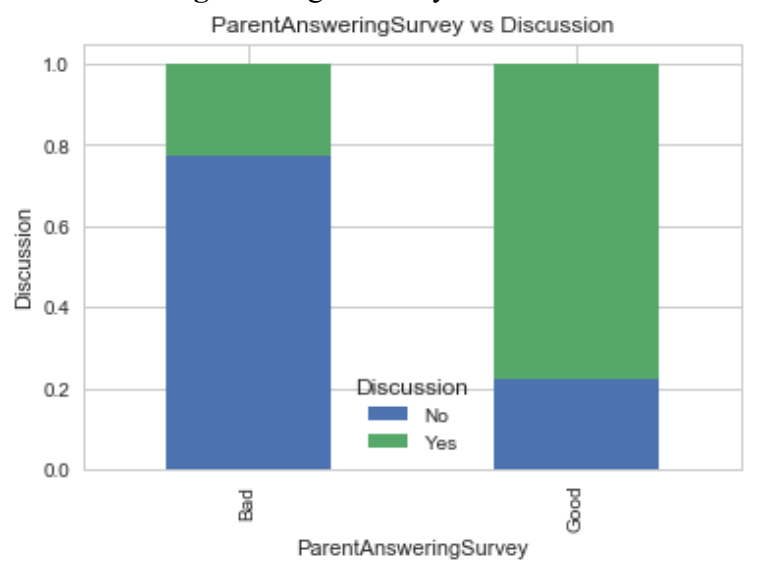

Fig 4 Discussion feature based Analysis

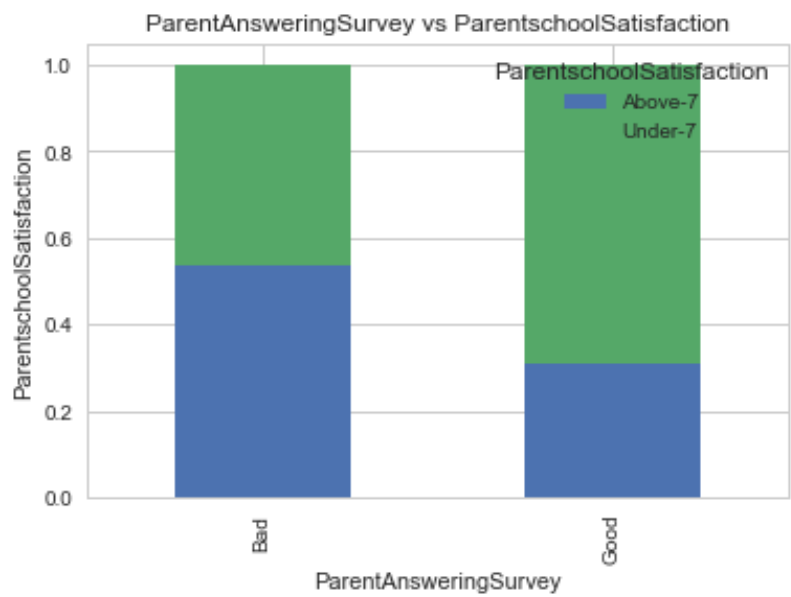

Fig 5 Satisfaction feature based Analysis 


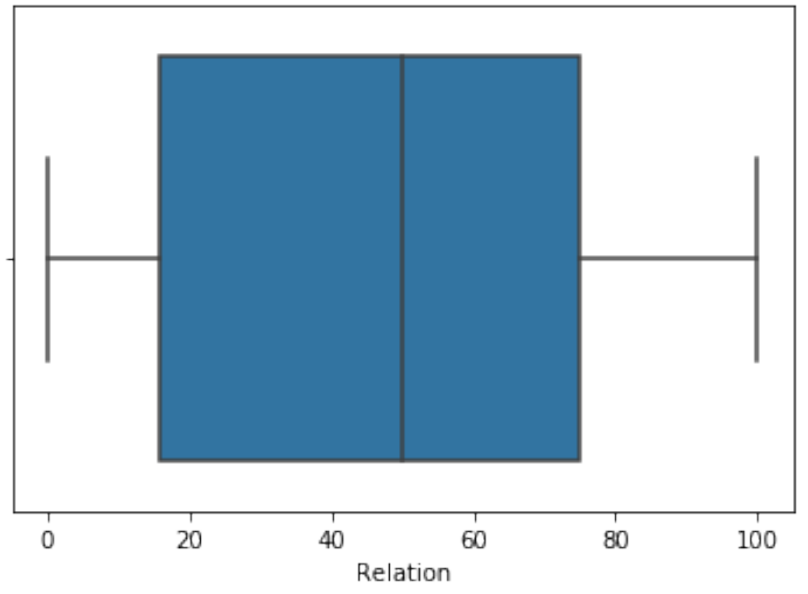

Fig 6 Relation density analysis

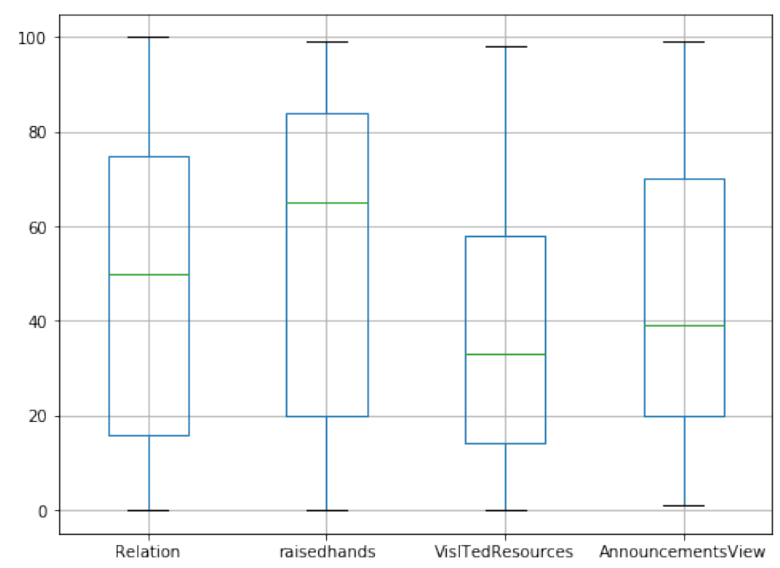

Fig 7 Relevant Feature Analysis 

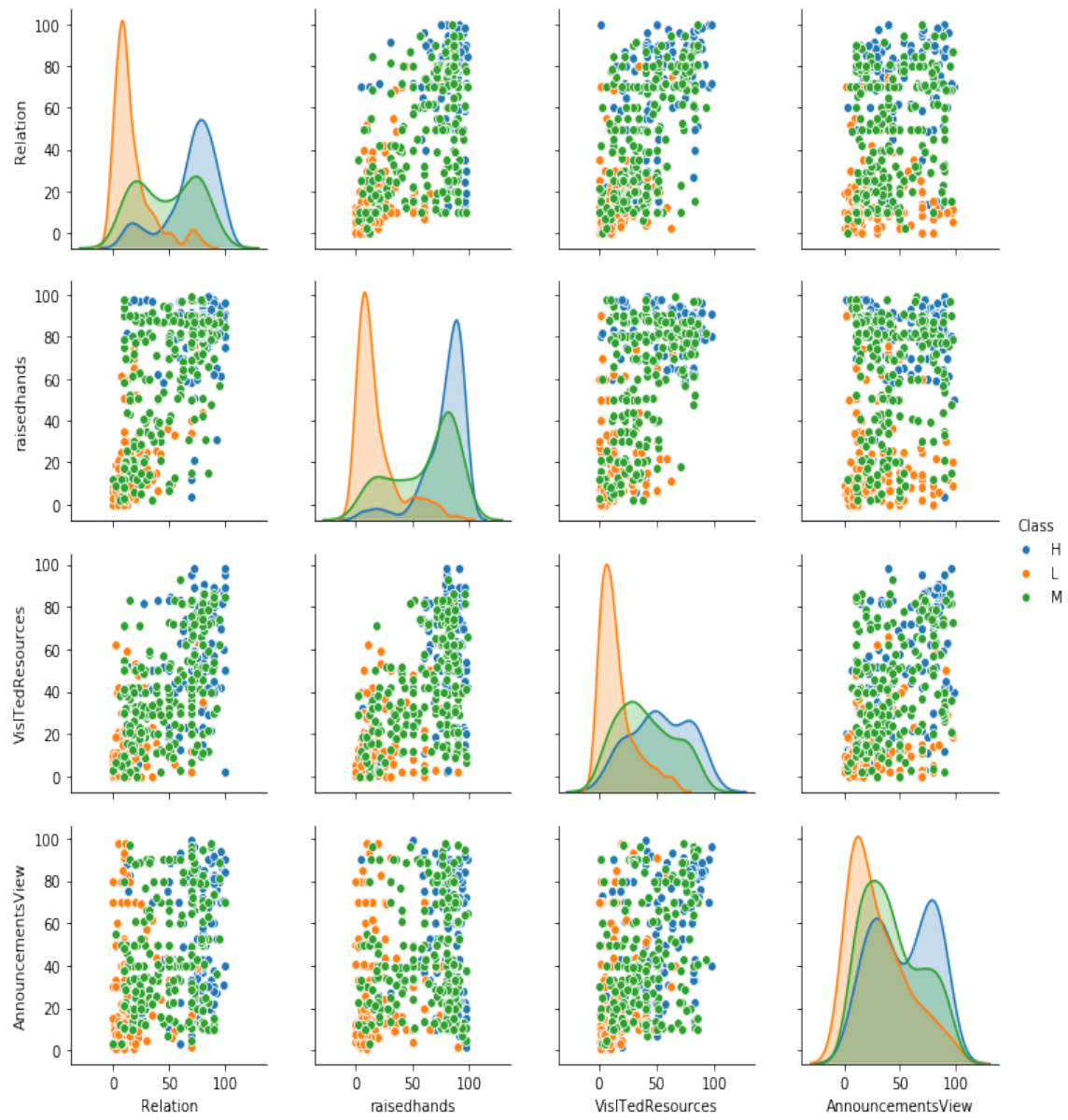

Fig 8 Review Relevancy Analysis 

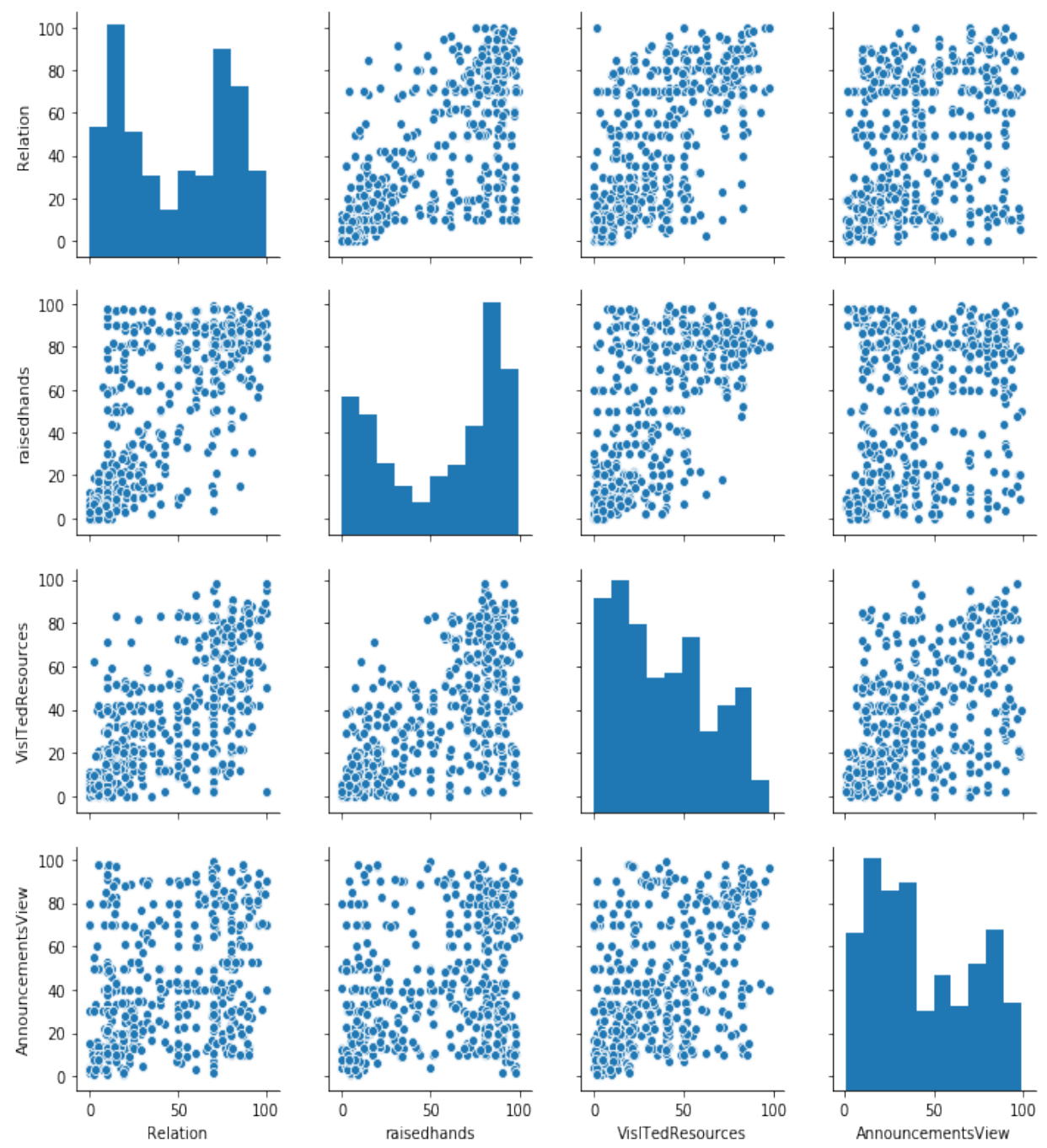

Fig 9 Review Classification Analysis

Table 3 Classification Summary for KNN

2. Classification report for KNN model: precision

$$
\text { recall f1-score support }
$$

$\begin{array}{rrrrr}\text { H } & 0.76 & 0.61 & 0.67 & 46 \\ \text { L } & 0.80 & 0.80 & 0.80 & 41 \\ \text { M } & 0.64 & 0.74 & 0.68 & 57 \\ & & & & \\ \text { accuracy } & & & 0.72 & 144 \\ \text { macro avg } & 0.73 & 0.72 & 0.72 & 144 \\ \text { weighted avg } & 0.72 & 0.72 & 0.72 & 144\end{array}$




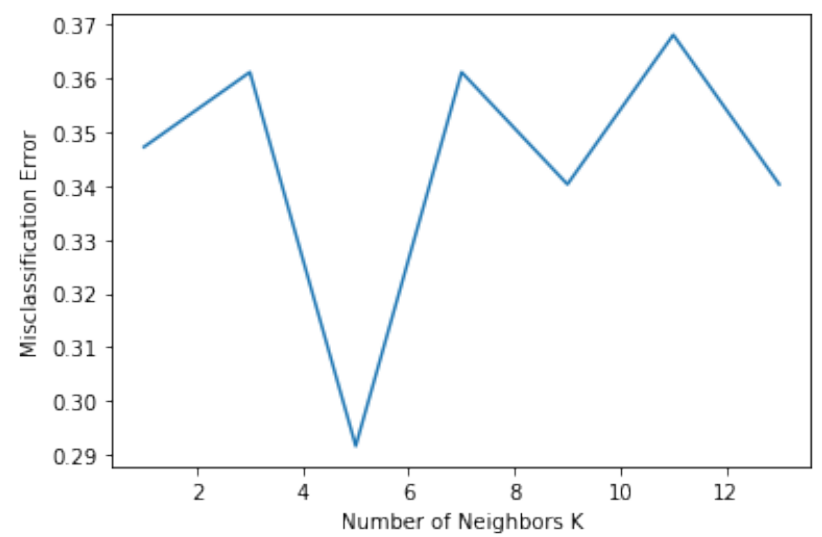

Fig 10 KNN Performance analysis

\section{Conclusion and future work}

Traditional review and recommendation system provides the information which is collected from the customer or user for evaluation. The feedback provides the complete quality of the particular domain because it is collected over various area such as education, marketing and so on. The specific framework does not support all kind of area, so the generic framework is needed for customized configuration. Various layers have been introduced for proper organization of the framework model. User interface layer support and generate the custom based access where as the feedback evaluation model collects various parameters, evaluate the parameters and submit the evaluation result for subsequent process. The configuration layer has been introduced for assigning and allocating suitable parameter with high quality. The final layer is a report generating layer which is used to view the evaluated result for performing decision making process. The main objective of the proposed generic review framework is to achieve the reliable result with high level of accuracy using KNN algorithm. In future this framework can be applied the IoT based system with real time application and its review process with high dimensional information.

\section{References}

[1]. Lakshmi Ramachandran, Edward F. Gehringer, "Automated Assessment of Review Quality Using Latent Semantic Analysis",2011 IEEE 11th International Conference on Advanced Learning Technologies, INSPEC Accession Number: 12191677 , DOI: 10.1109/ICALT.2011.46,2011, IEEE Pp.1-8.

[2]. Killian Muollo, Prateek Basavaraj, Ivan Garibay, "Understanding Students' Online Reviews to Improve College Experience and Graduation Rates of STEM Programs at the Largest Post-Secondary Institution: A Learner-Centered Study",2018 IEEE Frontiers in Education Conference (FIE),DOI: 10.1109/FIE.2018.8658450, IEEE, 2018, Pp.1-8.

[3]. Pawan Agarwal,Christopher D. Hundhausen,"A Socio-Psychological Approach to Improve Student Participation and Review Quality in Peer Code Reviews", 2010 IEEE Symposium on Visual Languages and Human-Centric Computing, IEEE, 2010, Pp.1-10. 
[4]. Irfan Prazina ; Vensada Okanović, "Methods for Double-Blind Peer Review and Grade Prediction of Student Software Projects",42nd International Convention on Information and Communication Technology, Electronics and Microelectronics (MIPRO),DOI: 10.23919/MIPRO.2019.8757122,IEEE, Pp 1-6.

[5]. Omid Mirmotahari ; Yngvar Berg ; Ester Fremstad ; Crina Damsa,"Student Engagement by Employing Student Peer Reviews with Criteria-Based Assessment", IEEE Global Engineering Education Conference (EDUCON),DOI: 10.1109/EDUCON.2019.8725174, IEEE, 2019,Pp1-8.

[6]. Pantelis M. Papadopoulos ; Thomas D. Lagkas, "Usage Data and Group Rankings in Peer Review Settings: A Case Study on Students' Behavior and Performance",IEEE 14th International Conference on Advanced Learning Technologies, DOI: 10.1109/ICALT.2014.92, IEEE, 2014, Pp 1-10.

[7]. Sayuri Yoshizawa ; Takao Terano ; Atsushi Yoshikawa, "Assessing the Impact of Student Peer Review in Writing Instruction by Using the Normalized Compression Distance", IEEE Transactions on Professional Communication ( Volume: 55 , Issue: 1, March 2012 ), DOI: 10.1109/TPC.2011.2172833,Pp.85 - 96.

[8]. Yang Chun, "Evaluation of College Students' Quality on PCA-BP Neural Networks", International Conference on E-Business and E-Government,2010, DOI: 10.1109/ICEE.2010.1005, IEEE,Pp 1.4.

[9]. Kay Berkling, Katja Neubehler, "Boosting Student Performance with Peer Reviews; Integration and Analysis of Peer Reviews in a Gamified Software Engineering Classroom",IEEE Global Engineering Education Conference (EDUCON),DOI: 10.1109/EDUCON.2019.8725247, IEEE, 2019.

[10]. Ai-Lun Wu ; Shun-Jyh Wu ; Shu-Ling Lin, "Grey relational analysis of students' behavior in LMS",International Conference on Machine Learning and Cybernetics,IEEE, 2011, Pp. 1-10.

[11]. M. Sivakumar, U. Srinivasulu Reddy, "Aspect based sentiment analysis of students opinion using machine learning techniques",International Conference on Inventive Computing and Informatics (ICICI),2017, DOI: 10.1109/ICICI.2017.8365231, IEEE, Pp. 1-8.

[12]. Samuel Cunningham-Nelson, Mahsa Baktashmotlagh, Wageeh Boles, "Visualizing Student Opinion Through Text Analysis", IEEE Transactions on Education,Volume: 62 , Issue: 4 , Nov. 2019, Pp.305-311

[13]. Jitendra Kumar Rout, Anmol Dalmia, "Revisiting Semi-Supervised Learning for Online Deceptive Review Detection", Special Section on Security and Privacy In Applications And Services For Future Internet Of Things, IEEE Access, Volume 5, 2017, Pp.1-9.

[14]. Thanh-Son Nguyen ; Hady W. Lauw ; Panayiotis Tsaparas, "Review Selection Using Micro-Reviews", IEEE Transactions on Knowledge and Data Engineering, Volume: 27 , Issue: 4 , April 1 2015,Pp 1098 - 1111.

[15]. Xu Yuan ; Mingyang Sun ; Zhikui Chen, "Semantic Clustering-Based Deep Hypergraph Model for Online Reviews Semantic Classification in Cyber-Physical-Social Systems", Cyber-Physical-Social Computing and Networking, IEEE Access, Volume: 6, Pp.17942 17951.

[16]. Pawan Lingras ; Matt Triff, "Fuzzy and Crisp Recursive Profiling of Online Reviewers and Businesses", IEEE Transactions on Fuzzy Systems, Volume: 23 , Issue: 4 , Aug. 2015,Pp 1242 - 1258.

[17]. M. Geetha, C.Saravanakumar, Ashwin Balakrishna, S.R.Nikitha Keerthana, An Efficient Multilevel Feature Extraction and Integrated Technique for Environment Landslide 
Prediction Using Machine Learning Technique, Journal of Green Engineering, volume10,issue 6, Pp. 3247-3263. 